\title{
Stress Analysis of a Pipeline as a Hydropower Plant Structural Element
}

\author{
Stefan Ćulafić1 ${ }^{*}$ - Taško Maneski² - Darko Bajić ${ }^{1}$ \\ 1University of Montenegro, Faculty of Mechanical Engineering, Montenegro \\ 2University of Belgrade, Faculty of Mechanical Engineering, Serbia
}

\begin{abstract}
This paper describes pipeline stress analysis, primarilybranch junctions, as a structural element in hydro-power plants. Pipelines are exposed to internal pressure,which is present under working conditions. Analysis of stresses in the pipeline of the hydropower plant is based on analytical, numerical, and experimental methods. In this paper, we will define the critical elements of the pipeline. After that, we will determine critical areas in the branch junction, under experimental conditions, where strain gauges should be installed. The obtained resultsshow that a boiler formula can be efficiently applied in the stress analysis. Also, a correlation between the internal pressure and the maximum circumferential stresses in the elastic zone is given. In the final sections of the paper, the limit value of the internal pressure as a load for which stress in the zone of plasticity appears and the safety factor of the branch junction in the exploitation conditions are determined. The contribution of this work is the unification and deepening of the topic related to the problem of the testing ofhydro-power structural elements. Keywords:pipeline, branch junction, hydropower plant, strength analysis, shell intersection

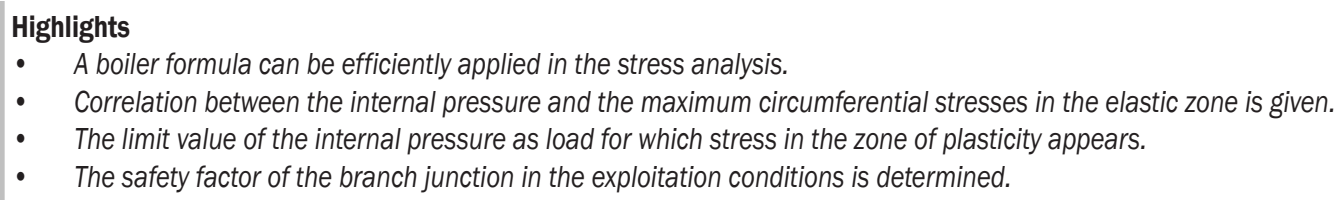

\section{O INTRODUCTION}

When studying hydropower plants, special attention should be paid to the analysis of the strength of certain parts of the hydropower plant. With a high-quality analysis of the stress of individual parts of the plant, it is possible, with sufficient accuracy in real conditions, to anticipate the critical areas for remediation, evaluation and reduction of the maintenance costs, which should ultimately extend the lifetime of the powerplant.

The interesting shell problem has not yet been investigated sufficiently. The practical importance of this problem requires further investigation, including that of the elastic stress analysis of the intersecting shells of the various shapes subjected to different loadings and the elastic-plastic analysis [1].

The geometry of hydropower plants' structural elements is very complex with large number of discontinuities; therefore, stress and strain analysis on these elements is also complex. Analytical analysis is possible only in a small number of very simple cases. Numerical analysis based on the finite element method is usedwhen analysing stresses of structural elements in pipelines, primarily pipeline branch junctions. It is a very real problem of defining locationsand areas of pipeline in which reinforcements should be made
[2] and also a problem of the estimation of limitin which yield stresses are observed [3], defining stress concentration factor, limit load [4] and [5], and burst pressure [6]. The use of experimental methods is very difficult under real, working conditions, because it is not possible to vary internal pressure in real conditions in some structural elements, such as branch junctions, until plastic strainsare observedunder working conditions. Because of this, it is more convenient to perform experiments on the model of the structural element under laboratory conditions and, on the basis ofthese results, make conclusions about what is happening in the real elements.

By combining numerical and experimental methods, it has been shown thatthe most accurate results are obtained when determining critical stresses, which (in some places) can lead to problems in the exploitation and functioning. The subject of this paper is the analysis of stress distribution in structural elements of a pipeline:

- applying analytical procedures,

- finite element method (FEM) application on the real element with real dimensions,

- FEM application on the element model,

- applying experimental analysis on the model under laboratory conditions. 


\section{METHODS}

Analytical, numerical, and experimental procedures were applied in the analysis of the stress of structural elements. One specific feature is in the fact that the experiment was realized not on a real structural element but on its model. Numerical analysis was also carried out on a real structural element and on a structural element model.

\section{ANALYTICAL AND NUMERICAL STRESS ANALYSIS OF PIPELINE}

The main characteristic of pipes in the pipeline is that their radius is much larger than the thickness $(R>>t)$, so it can be adopted that these pipes are actually shell pipeline.

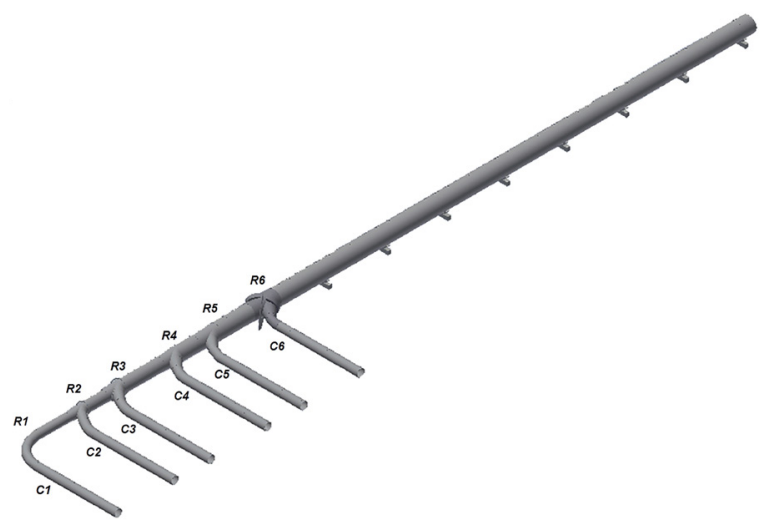

Fig. 1. FEM pipeline model

Static linear finite element analysisin the elasticity area was performed for the pipeline, i.e., its straight main tube parts and the knee part using Autodesk INVENTOR 2016 software. Due to a highly complex geometry,which has been analysed, the finite element (FE) analysis was used with caution, and also was confirmed with the KOMIPSstand-alone software package. FEM mesh was generated using 3D iso-parametric solid elements. A 3D model of the pipeline is shown in Fig. 1.The pipeline length is approximately $200 \mathrm{~m}$, and itis exposed to 51 bar of internal pressure under exploitation conditions. It can be remarked that the basic elements of the pipeline are branch junctions: R1,R2,R3,R4,R5,R6 (where branch junctions are actually knee pipes which direct the water)and pipes: $\mathrm{C} 1, \mathrm{C} 2, \mathrm{C} 3, \mathrm{C} 4, \mathrm{C} 5, \mathrm{C} 6$.

An analytical solution for the parts of the pipeline that is made of straight tubes with no junctions or nozzles is given. An analytical solution is also given for the pipeline knee (Fig. 2).
Analytical equations for the determination of stresses in the torus shell pipeline (Fig. 2)are known as follows:

$$
\begin{gathered}
\sigma^{o}=\frac{p \cdot R(2 a \pm R)}{2 t(a-R)}, \\
\sigma^{p}=\frac{p \cdot R}{2 t},
\end{gathered}
$$

where $p$ is internal pressure, $R$ radius of a circular cross-section, $a$ radius of a torus, $\sigma^{O}$ circumferential stress, and $\sigma^{p}$ longitudinal stress.

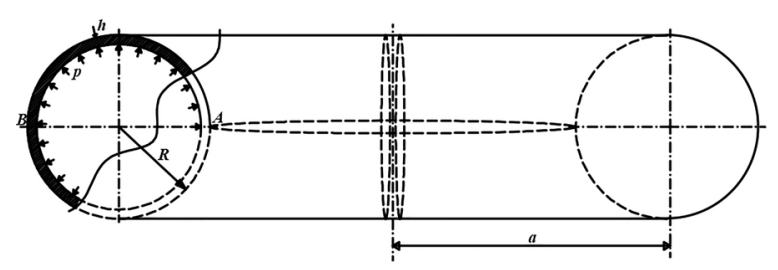

Fig. 2. Torus shell part of the pipeline

Especially, if $a=\infty$ it is a cylindrical shell, and if $a=0$ it is a spherical shell. On the basis of Eq. (1), the stresses can be calculated on all parts of the pipeline, except for branch junctions. It is thus possible to calculate the stresses on the kneepart R1 and the straight pipes of the pipeline $(\mathrm{C} 1, \ldots, \mathrm{C} 6)$.

Therefore, for points $\mathrm{A}$ and $\mathrm{B}$ of the knee $\mathrm{R} 1$ pipe ( $a=4650 \mathrm{~mm}, R=600 \mathrm{~mm}, t=18 \mathrm{~mm}, p=51$ bar): $\sigma_{A}^{O}=182.6 \mathrm{MPa}, \sigma_{B}^{O}=160.3 \mathrm{MPa}, \sigma^{p}=85 \mathrm{MPa}$, and for the pipes $\mathrm{C} 1$ and $\mathrm{C} 6$ : $\mathrm{C} 1: \sigma^{O}=170 \mathrm{MPa}, \sigma^{p}=85$ $\mathrm{MPa}, \mathrm{C} 6: \sigma^{O}=212.5 \mathrm{MPa}, \sigma^{p}=106.25 \mathrm{MPa}$.

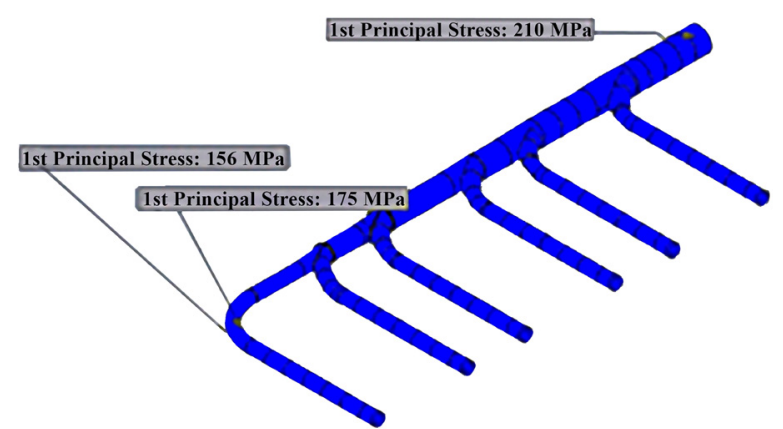

Fig. 3. FEM pipeline model

Since there wasone plane of symmetry, boundary conditions were the following: all translations normal to the plane of symmetry and all rotations in the plane of symmetry are constrained.

The material used in the FEM analysis was the most similar to thecharacteristics of NIOVAL 47, which are material properties of the pipeline under real working conditions. This is also important 
because of the correlation with the analytical results for whichYoung's elasticity modulus for steel has been used. A constant internal pressure of 51 bar was the implied load used in the finite element analysis.

Based on the numerical analysis, the stress values of which are given in Fig. 3, and analytical analysis, it can be seen that the pipeline branch junctions, especially branch junction number $\mathrm{C} 6$, are the most affected elements of the whole pipeline, so only branch junction number $\mathrm{C} 6$ will be the subject of further analysis.

\subsection{FEM Analysis of the Pipeline Branch Junction (Real Dimensions)}

Pipeline branch junctions (cylinder-to-cylinder intersections) are very often used in industrial engineering. Reduction of the base material due to penetration of the intersecting cylinder is the cause for stress concentration.

Authors [6] indicate that cylinder-to-cylinder intersections are a widespread occurrence in many industrial applications. Difficulties in obtaining analytical evaluations of the stress distributions in the disturbed regions near the intersection of comparably sized shells originally stemmed from the complicated geometrical shape of the intersection line. The intersection curve of the middle surfaces of the cylinders is neither rotationally symmetric, nor on a plane curve, but rather is a spatial curve. Moreover, the sharp discontinuities of curvatures across the intersection curve increase the stress.

Therefore, the presence of the stress concentration is inevitable and, as a consequence, constitutes a significant consideration in the design.

In paper [7] online monitoring proposition was given, and in papers [8] and [9] analysis of the pipeline branch junction in real dimensions wasperformed using FEM. The primary pipe diameter on the branch junction entrance is $2.5 \mathrm{~m}$, while pipe diameter at the exit is $2.35 \mathrm{~m}$. FEM was performed using AUTODESK Inventor 2016 software, in which we created the geometric model and performed the stressstrain analysis.

In Figs. 4 to 7, yield stresses are given when a pipeline branch junction in real dimensions is exposed to 20 bar, 50 bar and 84 bar, and 51 bar pressure.

Results of the FEM analysis shows that yield stress appears in the area next to the anchor of the pipeline branch junction. This particular area was specified (and referred to in Table 1 as MP1) as area of highest stress values. In Table 1, values of maximal circumferential stresses are given as a function of internal pressure values. Generally speaking, it can be said that thedependence of the value of stresses on pressure is very satisfactorylinear in the field of elastic strains, which further means that the branch junction, which is essentially a shell, is not loaded on bending.

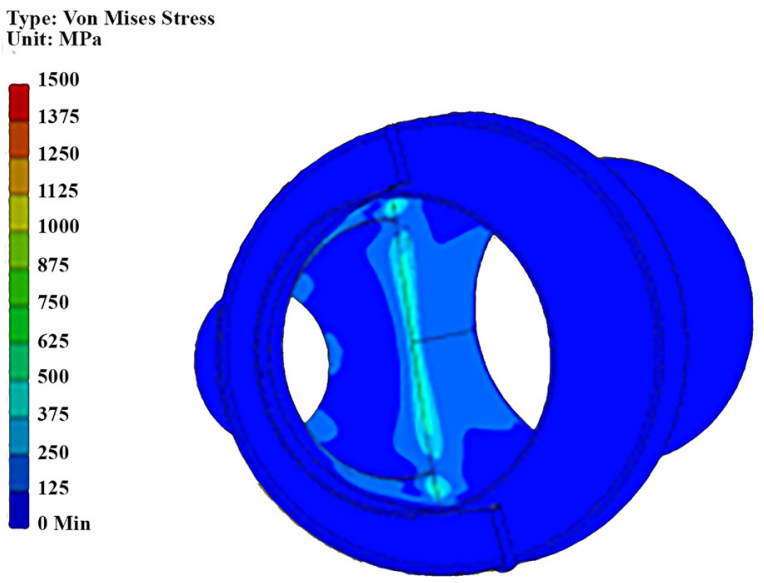

Fig. 4. FEM branch junction under 20 bar pressure

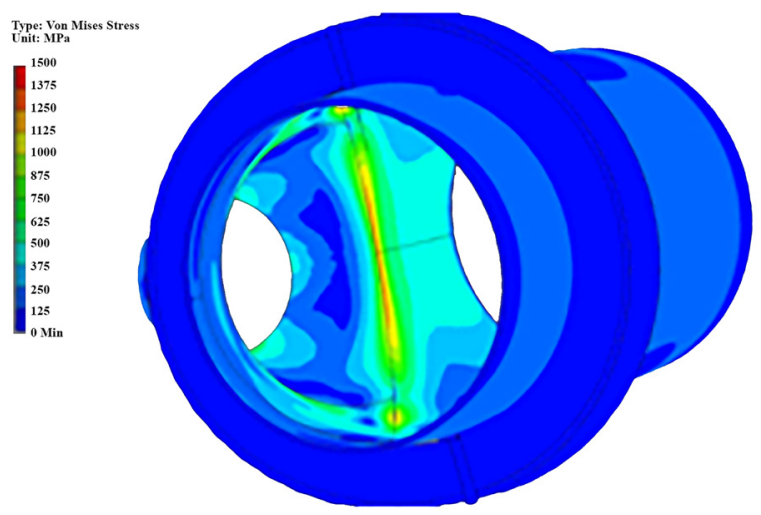

Fig. 5. FEM branch junction under 50 bar pressure

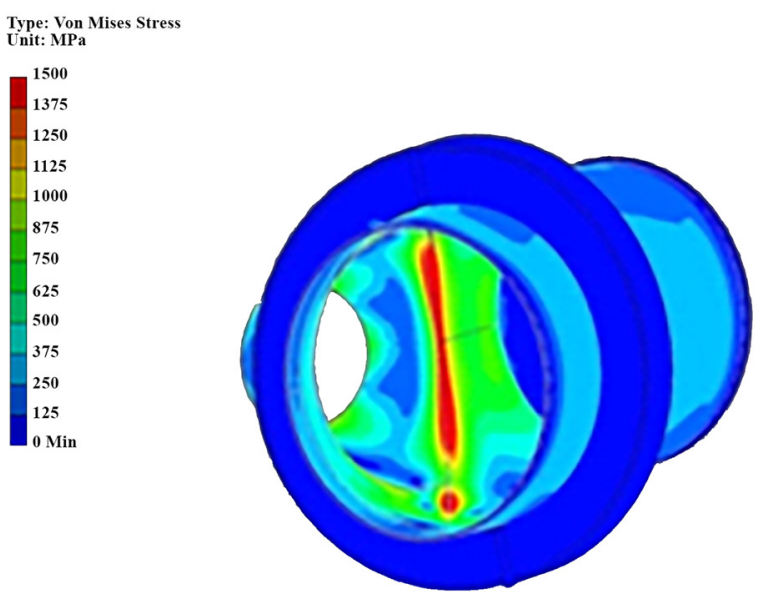

Fig. 6. FEM branch junction under 84 bar pressure 
Table 1. Stress values for MP1

\begin{tabular}{cc}
\hline Pressure values [bar] & Stress values for MP1 $[\mathrm{MPa}]$ \\
\hline 20 & 111 \\
\hline 50 & 284 \\
\hline 84 & 458 \\
\hline
\end{tabular}

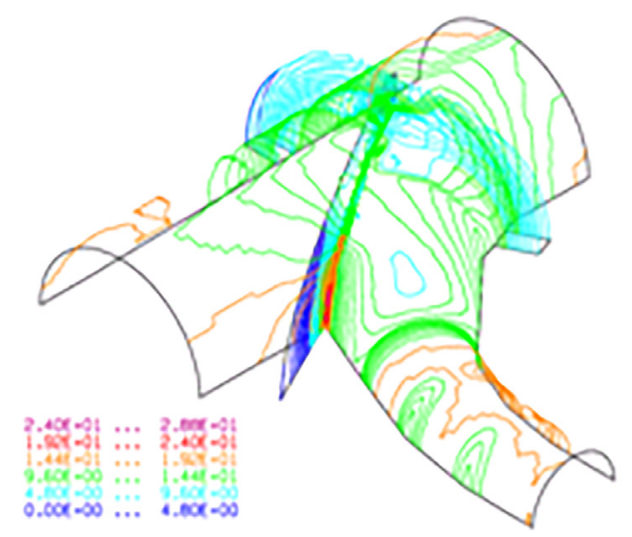

Fig. 7. Equivalent stress under 51 bar pressure

\section{EXPERIMENTAL}

\subsection{Branch Junction Model Manufacture}

The material used for the construction of pipe elements of the real object is NIOVAL 47, manufacturer SIJ - Slovenian Steel Group. The mechanical properties of this material are given in Table 2. In the absence of NIOVAL 47, which, due to exploitation problems, ceased to be produced during the 1970s, we analysed the steel of the same class with the most similar mechanical properties. That is steel S355J2 + AR. It was used to produce the branch junction model. The mechanical properties of this material are given in Table 3.

Table 2. Mechanical properties of the material NIOVAL 47

\begin{tabular}{ccc}
\hline $\begin{array}{c}\text { Tensile strength, } \\
R m[\mathrm{MPa}]\end{array}$ & $\begin{array}{c}\text { Yield strength, } \\
\operatorname{Re}[\mathrm{MPa}]\end{array}$ & $\begin{array}{c}\text { Elongation, } \\
A[\%]\end{array}$ \\
\hline 650 & 470 & 24 \\
\hline
\end{tabular}

Table 3. Mechanical properties of the material S355J2+AR

\begin{tabular}{ccc}
\hline $\begin{array}{c}\text { Tensile strength, } \\
R m[\mathrm{MPa}]\end{array}$ & $\begin{array}{c}\text { Yield strength, } \\
\operatorname{Re}[\mathrm{MPa}]\end{array}$ & $\begin{array}{c}\text { Elongation, } \\
A[\%]\end{array}$ \\
\hline 554 & 360 & 28.2 \\
\hline
\end{tabular}

Branch junction model (partitions Ø2500/Ø2350/ $\varnothing 1200 \mathrm{~mm}$ ) was made [10] of steel S355J2+AR, based on boiler formula. Branch model was created with the following characteristics:
- model dimensions are five times smaller than the real object,

- thickness is 10 times smaller.

Calculus per taining to stresses in the branch junction construction is analytically possible only in cylindrical parts of the junction, except for stiffeners, ribs and holes. The formula in which the stresses calculus is obtained is called the "boiler formula".

Boiler formula for stresses calculuson the cylinder (pipe, vessel) exposed to internal pressure without ribs and holes is Eq. (2) for the longitudinal direction of a cylinder and for:

- circumferential direction of a cylinder:

$$
\sigma^{o}=\frac{p \cdot R}{t}
$$

where $p$ is fluid internal pressure [bar], $R$ cylinder radius [mm] and $t$ cylinder thickness [mm].

From Eqs. (2) and (3), an observation can be made that values of the stresses in the circumferential direction are twice the value along the longitudinal direction.

A branch junction model should give the same stress as the real object has. This is secured by the application of similarity method in the following manner:

$$
\sigma^{o}=\frac{p \cdot R}{t}\left(=\frac{\frac{p}{2} \cdot \frac{R}{5}}{\frac{t}{10}}\right)=\frac{p_{\text {model }} \cdot R_{\text {model }}}{t_{\text {model }}} .
$$

Derivation of the same stress value is as follows: $p_{\text {model }}=p / 2, R_{\text {model }}=R / 5, t_{\text {model }}=\mathrm{t} / 10$.

Real branch junction: $p=50$ bar, $R=1250 \mathrm{~mm}$, $t=36 \mathrm{~mm}, \sigma^{o}=174 \mathrm{MPa},(R=34.77 \times t)$.

Branch junction model: $p=25$ bar, $R=250 \mathrm{~mm}$, $t=4 \mathrm{~mm}, \sigma^{o}=156 \mathrm{MPa}$.

This means that the branch junction model for thesame value of thepressure has twice the value of circumferential stresses. This also meansthe branch junction model has to be subjected to two times lower pressure so it could be correlated to the real branch.

In terms of manufacturing, we have adopted the previous relations. Since there was no $3.6 \mathrm{~mm}$ sheet, a $4 \mathrm{~mm}$ sheet was adopted. In this way, around 10 $\%$ smaller values of stresses are obtained. Anchor stresses are the same, since thicknesses are $8 \mathrm{~mm}$ forthe model and $80 \mathrm{~mm}$ forthe real branch.

Similarity method is applied on this branch junction model, because in its construction there is a negligible presence of bending stresses, as we concluded in the real branch analysis, which depends on a square $\left(t^{2}\right)$ of the thickness of the material. 


\subsection{FEM Analysis of Branch Junction Model}

A three-dimensional model of the branch junction of the basic dimensions and thicknesses is showninFig.8. It was made for the requirements of the strength analysis using FEM. Branch geometry was modelled using surfaces.

In numerical modelling, the branch is subjected to 10 bar of internal pressure on the walls. Due to linear static nature of the analysis of the construction (obtained stress has linear character compared to the given pressure), it is possible to use scaling to obtain results for different values of internal pressure. Taking into consideration the symmetry of the branch in longitudinal direction, one-half of the branch was analysed.

Mesh was more detailed in the areas of calotte penetration, where higher values of stress were expected. To control and confirm experimental analysis using strain gauges, FEM model was madeas well as necessary calculus.

Fig. 10 shows the positions of strain gauges on the branch. These exact locations will be usedfor the comparison of results between experimental tests and numerical results.

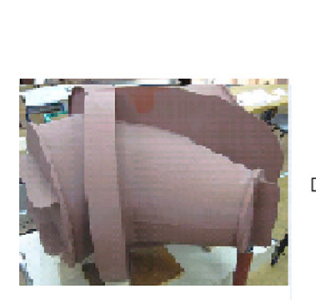

a)
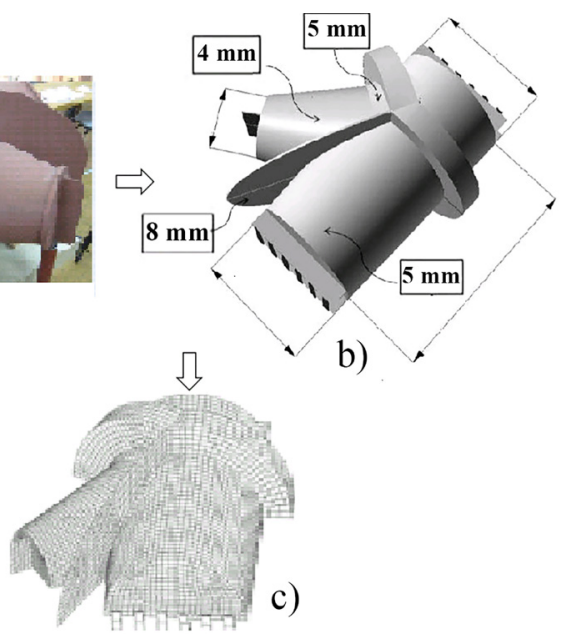

Fig. 8. Branch junction model; a) model in laboratory, b) FEM model, and c) mesh view

Boundary conditions are given as two constraints: translation and rotation, and since one plane of symmetry exists, the boundary conditions that were used were that all the nodes on the symmetric section were constrained against deformation in the perpendicular direction.

Fig. 9 shows the resultsof the FEM analysis as Von Mises yield stress and alsoas stresses in circumferential (vertical) direction. All results are related to the pressure of 10 bar. Also, Table 4 shows the exact values of circumferential stresses readings within the FEM model. These values are referred to as measuring positions MP1, MP2, and MP3.

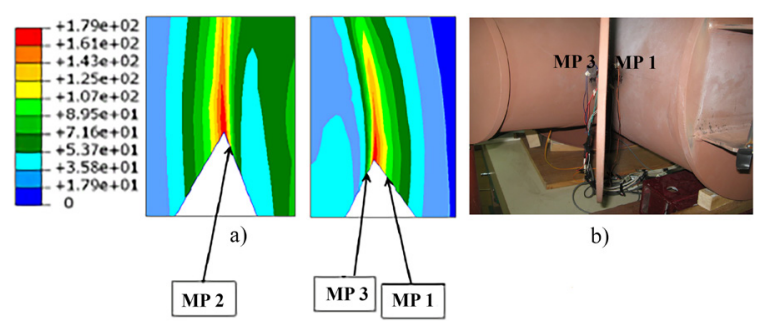

Fig. 9. Measuring positions of strain gauges; a) FEM view, and b) model view

Table 4. Values of stresses at measuring positions

\begin{tabular}{|l|c|c|c|}
\hline & MP1 & MP2 & MP3 \\
\hline Stress incircumferential direction [MPa] & 115 & 89 & 58 \\
\hline Von Mises stress [MPa] & 107 & 84 & 63 \\
\hline
\end{tabular}

The following conclusionscan be made based on the FEM analysis of the real branch junction and branch junction model:

1. The boiler formula gives, with very satisfactory accuracy, the correlation between the results on the real branch and branch model. This lies in the fact that the circumferential stresses on the MP1 are about the same, and amount to $111 \mathrm{MPa}$ on the real branch junction and $115 \mathrm{MPa}$ on the branch junction model. As the branch junction model is made on the basis of the boiler formula, we come to the previously stated conclusion. Furthermore, laboratory investigations have shown, based on the results obtained, what will happen with the branch junction under working conditions.

2. Yield stresses are visible in the area of the junctions of the main pipe $(\varnothing 2500 \mathrm{~mm})$ and anchor and branch pipe (Ø1200 $\mathrm{mm})$ and anchor, though it should be expected that measurements will show that the critical gauge position is position one.

\subsection{Experimental Analysis of Branch Junction Model}

Based on the results obtained using the FEM, it is possible to define areas at which strain gauges should be placed and, based on the similarity theory,it is possible to determinethe internal pressure in the real branch junction precisely before plastic strain occurs. 


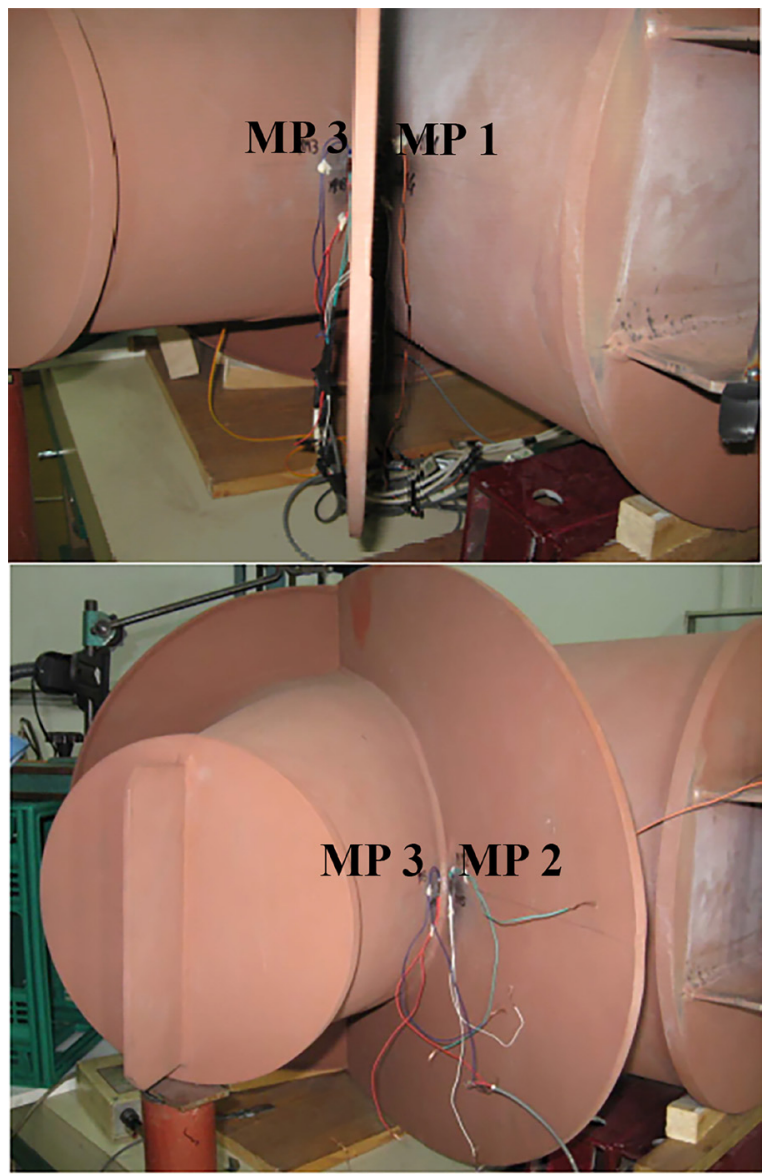

Fig. 10. Measuring positions: MP1, MP2, MP3

Measuring places are designated as follows: MP1, MP2, ..., MP8. Due to the complexity of the material, andobserved values of stresses in some measuring spots, focus of our attention will be onthe measuring positions MP1, MP2, MP3 (Fig. 10) where MP1 is acircumferential direction of bigger cylinder, MP2 a vertical direction of anchor, and MP3 a circumferential direction of the conical part of the model.

Numerical analysis of the branch junction in real conditions and the branch junction model have shown that biggest stresses are in MP1 (Tables 3 and 4).

Twenty-three experiments were conducted for each measuring position.

Values of the stress for any value of the pressure are obtained when scaling these values by a given factor.

In the following Figs. 11 and 12 graphical representation of measured stresses in all measuring positions and experiments conducted are givenas the function of pressure and time.

It can be seen from Figs. 11 and 12 how the stresses change at all measuring positions with the change of internal pressure. At the pressure of 20 bar, the value of the stress on MP1 is slightly higher than $200 \mathrm{MPa}$. It is also noted that the MP1 stress is dominant in relation to the stresses at other measuring positions. After that, the highest stress value is on MP2. Also, it should be noted that after unloading, the internal pressure returns to the initial, i.e.,zero, and that this release is accompanied by the stresses that also return to zero. This further means that, as far as the stress condition is concerned, the process is related to elasticity, i.e., stresses do not reach the value that belongs to plastic strain beyond the yield point.

Table 5 shows mean values based on all experiments at MP1,MP2,MP3, when pressure is reduced to 10 bar.

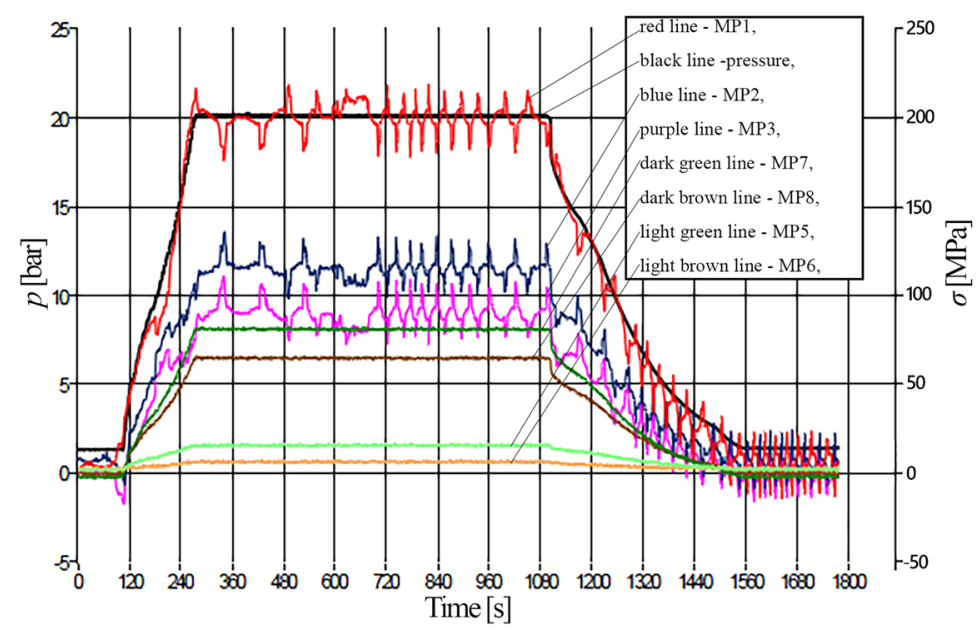

Fig. 11. Internal pressure and stress as the function of time (elastic strain) 


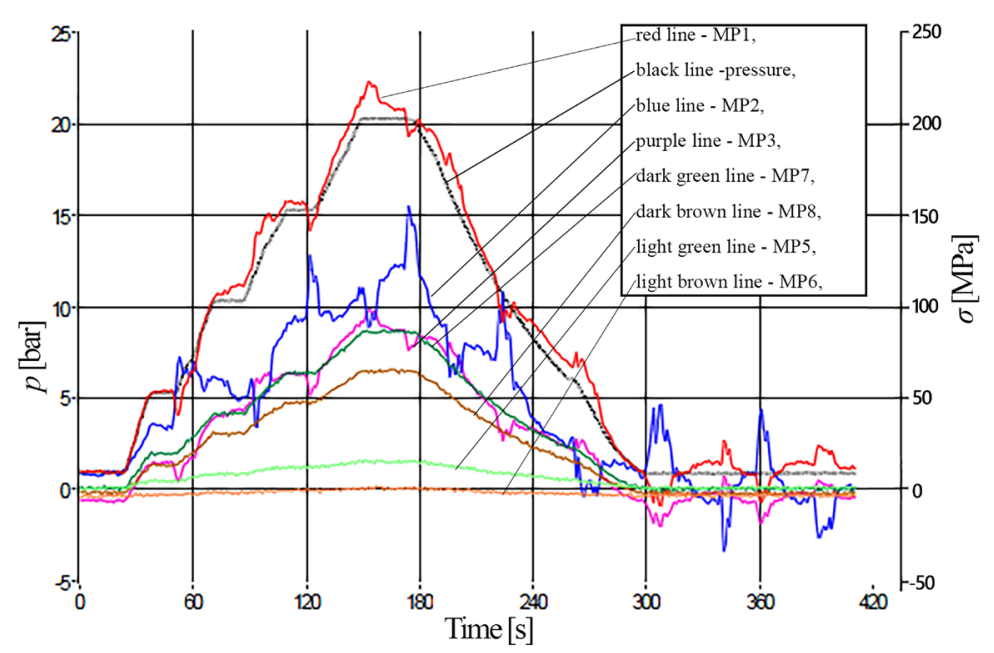

Fig. 12. Internal pressure and stress as the function of time (elastic strain)

Table 5. Mean values of stress on measuring positions (MP)

\begin{tabular}{cc}
\hline MP & Pressure value $[\mathrm{MPa}]$ under 10 bar \\
\hline MP1 & 110 \\
\hline MP2 & 75 \\
\hline MP3 & 50 \\
\hline
\end{tabular}

This scaling only applies until yield point and enables the acquisition of any value of pressure stresses.

\section{RESULTS}

\subsection{Comparison of the Measurements and the FEM Calculation}

Table 6 gives values of stress intensities for the positions MP1, MP2, MP3 at the branch junction model obtained when the FEM calculation is applied and when measuring values (for 10 bar) were obtained [11]. The general conclusion is that these values are very close, which puts these two methods in equal position and simultaneously confirms each other.

Table 6. Mean values of stresses at MP1, MP2, MP3

\begin{tabular}{ccc}
\hline \multirow{2}{*}{ MP } & \multicolumn{2}{c}{ Stress [MPa] } \\
\cline { 2 - 3 } & FEM & Measured \\
\hline MP1 & 115 & 110 \\
\hline MP2 & 89 & 75 \\
\hline MP3 & 58 & 50 \\
\hline
\end{tabular}

\subsection{Determination of Limit Internal Pressure}

When the pressure is increased to 30 bar, the stress on MP1 reaches a value of about $370 \mathrm{MPa}$ (Fig. 13). It is also important to note that after unloading, i.e. bringing the internal pressure to zero, the stress value on MP1 does not return to zero, but has a value of $50 \mathrm{MPa}$. This means that there is a permanent deformation corresponding to this stress. When, after the permanent deformation, the branch junction model is again submitted to the pressure of 30 bar, the stresses at MP1, after unloading return to the new starting level of $50 \mathrm{MPa}$.

From Fig. 14, it can be seen that when the pressure is increased to 32 bar to 33 bar, additional permanent deformation of the branch model at MP1 is made, for additional $50 \mathrm{MPa}$. When the branch model is unloaded, the stress at MP1 is $100 \mathrm{MPa}$. Fig. 15 shows the behaviour of the stress on the MP1 model of the branch when the internal pressure reaches the value of 40 bar to 45 bar. It can be seen that the stress value at MP1 reaches $850 \mathrm{MPa}$.

The pressure of 45 bar on the branch junction model corresponds to 90 bar on the real branch junction. From the diagram shown in Fig. 15, it can be seen that the stress value is about $850 \mathrm{MPa}$. If we deduct from this value the value of the stress due to the plastic strain of about $350 \mathrm{MPa}$, we obtain the value of about $500 \mathrm{MPa}$, which corresponds to the internal pressure of 90 bar on the real branch or 45 bar on the branch model. This further means that the result given in Table 1 is logical (pressure of 42 bar on the branch model or 84 bar on the real branch) and corresponds 


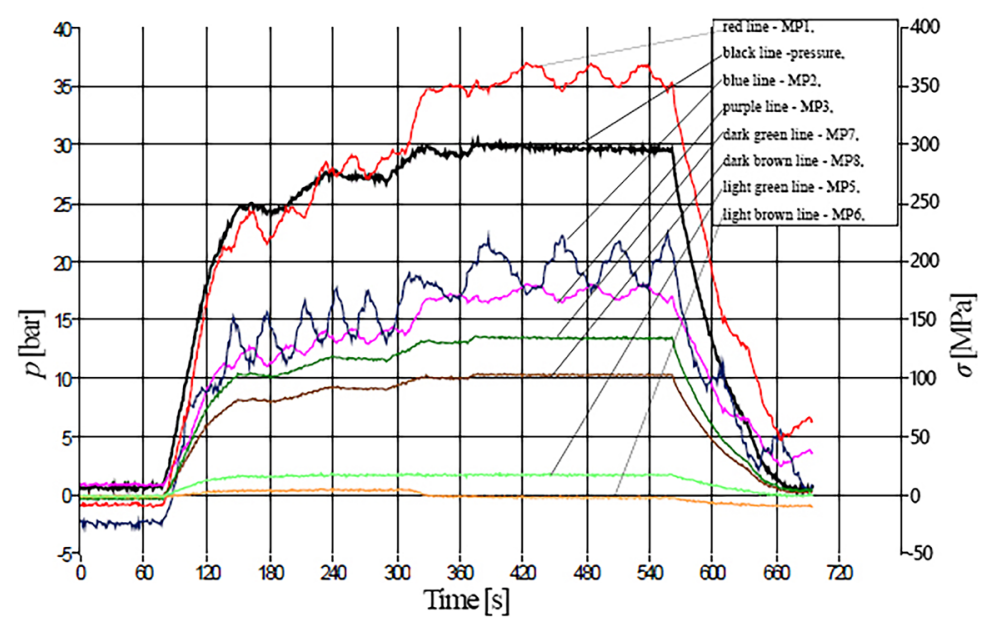

Fig. 13. Internal pressure and stress as the function of time (plastic strain)

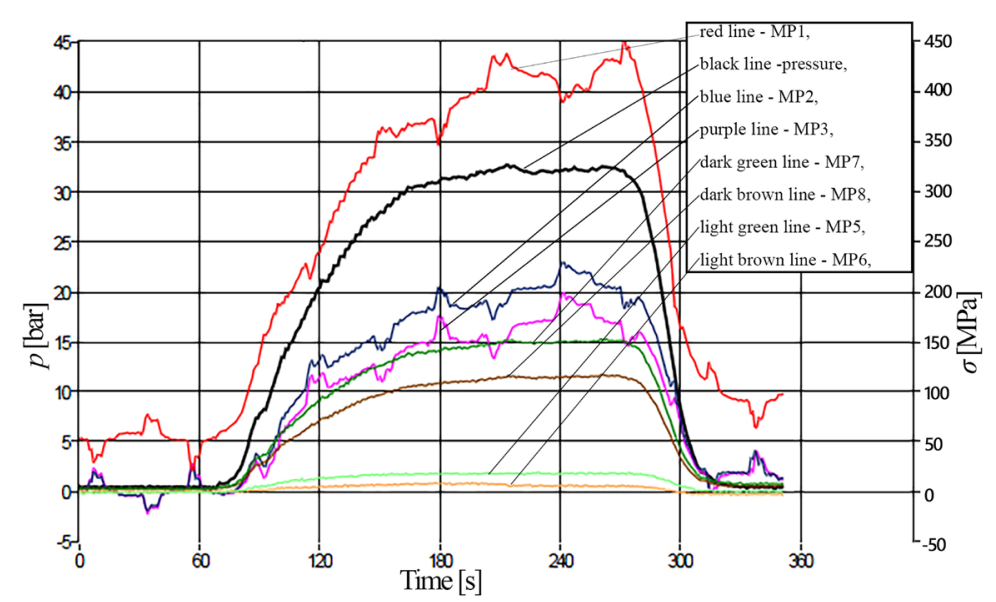

Fig. 14. Internal pressure and stress as the function of time (plastic strain)

to the maximum stress value of $458 \mathrm{MPa}$ at MP1.The explanation lies in the fact that the results given in Table 1 are related to the assumption that all analyses based on the finite element method are related to the field of elasticity.

\subsection{Determination of Safety Factor}

In the paper [12] a procedure for the determination of the stress concentration factorwas given. As the continuation of this paper, we will determine the safety factor.

Initial plastic strains of the branch junction model appear on MP1 under the pressure of 30 bar.

Calculation of necessary pressure for the occurrence of the initial plastic strains on the real branch junction on the measuring position MP1 (position of maximum stress) is as follows:

$$
P=30 \text { bar } \times 0.9 \times(47 / 36) \times 2=70.5 \text { bar. }
$$

Factor 0.9 represents the relation of the thickness of the real branch junction and branch junction model on the position MP1 $36 /(4 \times 10)=0.9$. Relation $47 / 36$ represents the relation of yield point of the material of the branch (NIOVAL 47) and of the branch model ( $\mathrm{St355J} / \mathrm{AR}$ ). Factor 2 is model factor, which refers to the pressure.

The safety factor in the branch junction exploitation in relation to the plastic strains is $70.5 / 51$ $=1.38$.

From Fig. 15 (blue line, MP2), it can be seen that the first plastic strains on MP2 appear when the pressure reaches 45 barcorresponding to the stress of $70 \mathrm{MPa}$ to $80 \mathrm{MPa}$. If the pressure of $45 \mathrm{bar}$ is reduced by 5 bar, we conclude that at the pressure is about 40 bar, the first plastic strains appear. 


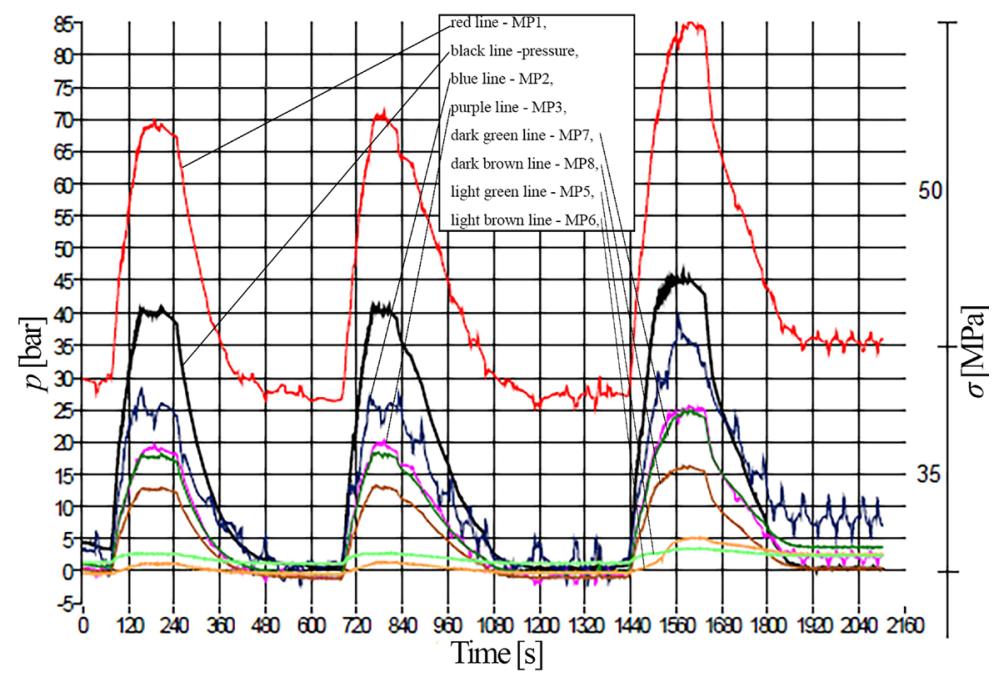

Fig. 15. Internal pressure and stress as the function of time (plastic strain)

Calculation of necessary pressure for the appearance of initial plastic strain on the real object of the pipeline branch junction on MP2 is:

$$
p=40 \text { bar } \times(47 / 36) \times 2=104.44 \text { bar. }
$$

Stress values on other measuring positions are even lower.

\section{CONCLUSION}

This paper attempts to use analytical, numerical, and experimental methods to describe the problem of the stress analysis inthe pipeline. It is shown that thecritical structural element of the pipeline is the pipeline branch junction. The following analyses have been carried out:

- Analytical and numerical analysis for the pipes of the pipeline and thekneesection of the pipeline;

- Numerical analysis of the branch junction in real dimensions;

- Numerical analysis of the branch junction model;

- Experimental analysis of the branch junction model.

Based on Eqs. (2) and (3), we defined the measuring places where the strain gauges should be placed. Also, it is shown that the boiler formula is correct for loads in which the plastic strain zone is not reached. Our paper also shows the linear correlation betweeninternal pressure andmaximum circumferential stress, which means thatbending stressescan be neglected, i.e. the branch junction can be treated and observed as a membraneshell. In case of appearance of the trapped air pockets, pressure will become unsteady [13] and [14]. This paper analysis only steady pressure.

Based on the experimental analysis of the branch junction model, it can be concluded how a real-dimension branch junction will behave under internal pressure, under real working conditions. It was shown that the results pertaining to obtaining stresses with numerical method on the real branch junction, results pertaining to obtaining stresses with numerical method on the branch model junction and results pertaining to the experimental determination of stress values are such that satisfactory accuracy has been reached, as it can be seen from Tables 4 and 6. Also, based on the experimental analysis, the limit pressure value was defined based on the point at which the first plastic strain is noted, which enabled us to define the maximum value of pressure under working conditions on the real branch junction necessary to achieve plastic strain (70.5 MPa). Thisenabled us to define the safety factor (1.38) in the branch junction exploitation as well as for the whole pipeline.

\section{REFERENCES}

[1] Skopinsky, V.N. (1993). Numerical stress analysis of intersecting cylindrical shells. Journal of Pressure Vessel Technology, vol. 115, no. 3, p. 275-282, DOI:10.1115/1.2929528.

[2] Barry, R.M., Venter, G. (2019). Analysis of reinforcement designs for specials in steel pipelines. International Journal of Pressure Vessels and Piping, vol. 169, p. 204-214, Dol:10.1016/j.ijpvp.2018.12.005.

[3] Kim, S.-H., Gim, J.-M., Wang, M., Kim, Y.J. (2016) .Simplified limit load estimation using mo-tangent method for branch pipe junctions under internal pressure and in-plane 
bending. Procedia Structural Integrity, vol. 2, p. 2583-2590, DOI:10.1016/j.prostr.2016.06.323.

[4] Plancq, D., Berton, M.N. (1998). Limit analysis based on elastic compensation method of branch pipe tee connection under internal pressure and out-of-plane moment loading. International Journal of Pressure Vessels and Piping, vol. 75, no. 11, p. 819-825, D0l:10.1016/S0308-0161(98)00085-4.

[5] Kim, Y.-J., Lee, K.-H., Park, C.-Y. (2008). Limit loads for piping branch junctions under internal pressure and in-plane bending- extended solutions. International Journal of Pressure Vessels and Piping, vol. 85, no. 6, p. 360-367, D0l:10.1016/j. ijpvp.2007.11.007.

[6] Sang, Z.F., Xue, L.P., Lin, Y.J., Widera, G.E.O. (2002). Limit and burst pressures for a cylindrical shell intersection with intermediate diameter ratio. International Journal of Pressure Vessels and Piping, vol. 79, no. 5, p. 341-349, Dol:10.1016/ S0308-0161(02)00028-5.

[7] Sedmak, A. (2011). Program for an On-Line Monitoring of Metal State in Pipeline no. 3 at HPP Perucica. Faculty of Mechanical Engineering, Podgorica.

[8] Culafic, S. (2015). Numerical and experimental analysis of stress state in pipeline branch in HP Perucica. MSc. thesis, Faculty of Mechanical Engineering faculty, Podgorica.
[9] Ćulafić, S., Bajić, D., Maneski, T. (2015). Numerical model of pipeline branch in HP Perucica. Proceedings of the $2^{\mathrm{ND}}$ International Conference onNew Technologies, p. 27-32.

[10] Bajic, D., Maneski, T. (2011). Investigation of Branch Junction A6 Pipeline no. 3. at HPP Perucica. Faculty of Mechanical Engineering, Podgorica.

[11] Bajić, D., Ćulafić, S. (2017). Comparison of Numerical and Experimental Results of Stress-Deformation State in a Pipeline Branch. Scientific Proceedings XIV International Congress on Machines, Technologies, Materials, p. 29-32.

[12] Bajić, D., Momčilović, N., Maneski, T., Balać, M.,Kozak, D., Culafić, S. (2017). Numerical and experimental determination of stress concentration factor for a pipe branch model. Tehnički vjesnik - Technical Gazette, vol. 24, no. 3, p. 687692, DOl:10.17559/TV-20151126222916.

[13] Bergant, A., Tijsseling, A., Kim, Y.l., Karadžić, U., Zhou, L., Lambert, M., Simpson, A. (2018). Unsteady pressures influenced by trapped air pockets in water-filled pipelines. Strojniški vestnik - Journal of Mechanical Engineering, vol. 64, no. 9, p. 501-512, D0I:10.5545/sv-jme.2018.5238.

[14] Bergant, A., Karadžić, U., Tijsseling, A.S. (2016). Dynamic water behavior due to one trapped air pocket in a laboratory pipeline apparatus. IOP Conference Series: Earth and Environmental Science, vol. 49, p. 967-976, D0I:10.1088/17551315/49/5/052007. 